\title{
A Checklist of Angiospermic Flora of Tinjure-Milke-Jaljale, Eastern Nepal
}

\author{
Dilkumar Limbu' ${ }^{1}$, Madan Koirala ${ }^{2}$ and Zhanhuan Shang ${ }^{3}$ \\ ${ }^{1}$ Central Campus of Technology \\ Tribhuvan University, Hattisar, Dharan \\ ${ }^{2}$ Central Department of Environmental Science \\ Tribhuvan University, Kirtipur, Kathmandu \\ ${ }^{3}$ International Centre for Tibetan Plateau Ecosystem Management \\ Lanzhou University, China \\ e-mail:dilklimbu@yahoo.com
}

\begin{abstract}
Tinjure-Milke-Jaljale (TMJ) area, the largest Rhododendron arboreum forest in the world, an emerging tourist area and located North-East part of Nepal. A total of 326 species belonging to 83 families and 219 genera of angiospermic plants have been documented from this area. The largest families are Ericaceae (36 species) and Asteraceae (22 genera). Similarly, the largest and dominant genus was Rhododendron (26 species) in the area. There were 178 herbs, 67 shrubs, 62 trees, 15 climbers and other 4 species of sub-alpine and temperate plants. The paper has attempted to list the plants with their habits and habitats.
\end{abstract}

Key words: alpine, angiospermic flora, conservation, rhododendron Tinjure-Milke-Jaljale

\section{Introduction}

The area of Tinjure-Milke-Jaljale (TMJ) falls under the middle Himalaya ranging from $1700 \mathrm{~m}$ asl to $5000 \mathrm{~m}$ asl, and geographically lies between $27^{\circ} 6^{\prime} 57^{\prime \prime}$ to $27^{0} 30^{\prime} 28^{\prime \prime}$ north latitude and $87^{\circ} 19^{\prime} 46^{\prime \prime}$ to $87^{\circ} 38^{\prime} 14^{\prime \prime}$ east longitude. It covers an area of more than $585 \mathrm{~km}^{2}$ of three districts, Sankhuwasabha, Tehrathum and Taplejung in the Eastern Development Region of Nepal (EDRN) (Fig. 1). It is one of the longest mountain ridges in between the watersheds of Arun and Tamor rivers, running through the boarder of the three districts. It is important to note that TMJ area lies approximately between the highest peak of the world (Mount Everest) and the lowest point (Kechana) of the country. The aerial distance from Mt. Everest (8848 m) to Milke Danda is $97 \mathrm{~km}$ and from Kechana (70 m) is $110 \mathrm{~km}$ (IUCN 2002).

TMJ has different climates from warm temperate in the lower part to alpine in the upper hill slopes, which determines overall biodiversity and development activities. With the increasing altitude, temperature is decreased and consequently different climatic zones within a sort vertical distance are found. The precipitation varies from 1000 to $2400 \mathrm{~mm}$, and the average is about $1650 \mathrm{~mm}$ over the TMJ region. With the increasing altitude, rainfall is increased within the short vertical distance.

Land form range from moderately sloping, steeply sloping to very steeply sloping. Moderately sloping land is about $40 \%$ of the total area suitable for crop cultivation and human settlement. Forest area is more in the steeply sloping land. Forest is the largest category in terms of land-use $59 \%\left(340.13 \mathrm{~km}^{2}\right)$ of the total land in TMJ which is followed by cultivated land occupies 24\% (140.97 km²). The share of bush/ shrub and grassland covers is $5 \%$ and $11 \%$ respectively. Other land viz. landslide and ponds cover only $0.4 \%$ of the total area (IUCN 2002). 


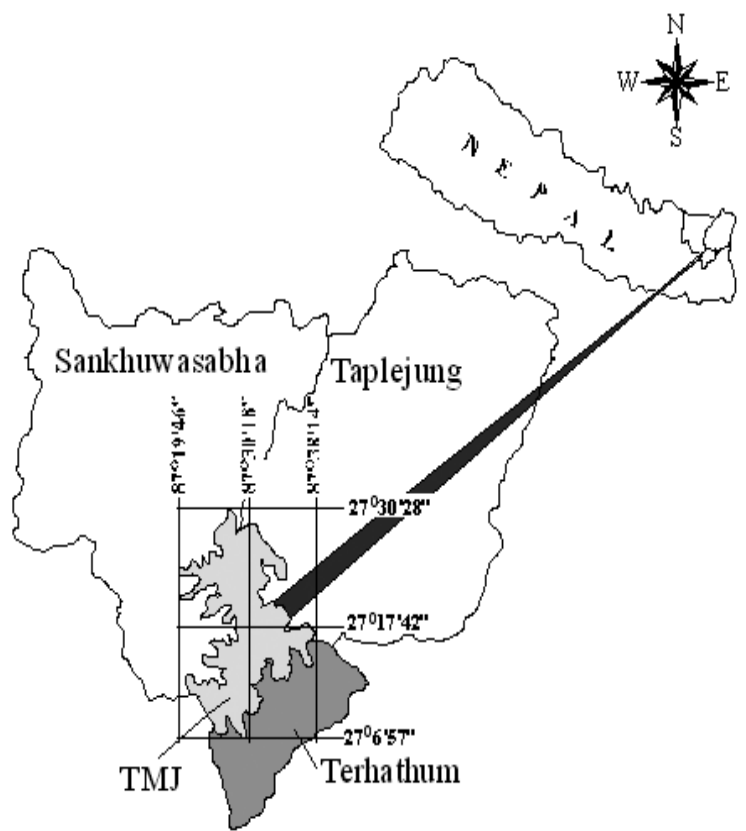

Fig. 1. Map of the study area, TMJ

TMJ area is the biodiversity treasure as well as considered as one of the important biological hotspot site of Nepal. It has high angiospermic floral diversity having 216 species according to IUCN, (2002) and 244 species according to Koiral a \& Limbu (2010). Similarly, it is considered as a biological corridor of Makalu Barun National Park and Kanchenjunga Conservation Area. The mountain ridges across TMJ are the natural niches for dozens of rhododendron species (Limbu, 2011). It is the home to 25 rhododendron species out of 30 species from Nepal (Chaudhary \& Taylor 2000) that is why it is called the capital of rhododendron in Nepal. TMJ is known to host the greatest rhododendron occurrence in Nepal. The Rhododendron arboreum forests of Milke Danda is arguably the largest rhododendron forest in the world (Milleville 2002). Similarly, the site is rich in birds and mammalian fauna and considered as a breeding ground to endemic birds. Thus, the primary aim of this work was to make a checklist of angiospermic flora of TMJ area.

The conservation area of the TMJ area was formally initiated in the mid 1990s when IUCN Nepal launched natural resource management program in TMJ area. The Government of Nepal declared TMJ area as National Rhododendron Environmental Conservation Area (NRECA) under the Ministry of
Population and Environment in 1997 (MOPE 1997). IUCN has developed infrastructure and enhanced local level social awareness activities which are most essential for conservation. At present, IUCN completed 10 years project duration and handed over to Nepal government and Nepal Government did not take further initiation and allocation of budget for development of this area. Thus entire programmes are in standstill.

\section{Methodology}

The paper is based on the species collected during September 2010 and May 2011. Information was collected by making regular field visits and species were identified by using available literature (Watson et al. 2011, Press et al. 2000, Hara et al. 1978, 1982, Polunin \& Stainton 2003, Stainton 2003, Hara \& Williams 1979, The Plant List 2010, version 1 ). Plant collection route was Basantapur $(2250 \mathrm{~m})$, Tute Deurali (2475m), Ghurbise (2660m), Pancha Pokhari (2850m), Tinjure (2900m), Phedi (267m), Chouki (2690m), Mangalabare (2635m), Shreemane, Bhalukhop, Lampokhari (2800m), Gupha (2890m), Jorpokhari (2905m), Milke (3016m), Lampokhari (Thadelung) (2880m), Khoping (3000m), Harkate (3200m), Dobate (3450m), Gorujure (3600m), Hile, Gidde, Manebhanjyang (3400m), Khambole (3500m), Gurgutte (Tree line 3850m asl), Thumki (3950m), Bhakari Chhiruwa (4000m) and Jaljale Pokhari (4100m asl). Attempt was made to enumerate the family, genera and species in alphabetical order with botanical names followed by local name, habit and habitat and presented in a tabular form. In case of collection and estimation of floral diversity of TMJ area, the allocated time period was short which did not allow to collect entire plant diversity. However, plant species were collected by the process of “Rapid Biological Assessments” (Primack 1998).

\section{Results and Discussion}

A total of 326 species belonging to 83 families and 219 genera of angiospermic plants have been documented from the TMJ proposed conservation area (Table 1). Out of these, 261 species belong to 70 families and 172 genera of dicotyledons whereas 65 species and 47 genera of 13 families of monocotyledons (Fig. 3). The largest families are Ericaceae (36 species), Asteraceae (30 species), Rosaceae (20 species), Poaceae (18 species), 
Cyperaceae (10 species), Polygonaceae (9 species), Lamiaceae and Urticaceae (8 species) and Araceae, Lauraceae and Orchidaceae (7 species). On the basis of genera, the largest families are Asteraceae (22 genera), Poaceae (18 genera) and Rosaceae (11 genera). Similarly, the largest genera are Rhododendron (26 species), Arisaema and Carex (6 species), Rubus (5 species) and Quercus, Anaphalis, Berberis and Persicaris (4 species).

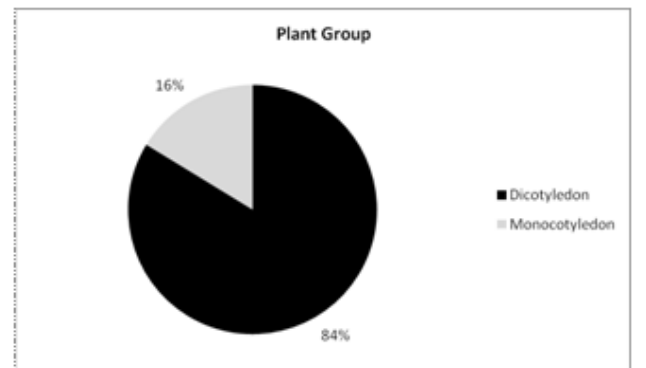

Fig. 3. Graphical representation of Plant's group of TMJ

Table. 1. Angiospermic flora of Tinjure-Milke-Jaljale area

\begin{tabular}{|c|c|c|c|}
\hline Family Name & Scientific Name & Local Name & Habit \\
\hline Dicotyledon & & & \\
\hline Aceraceae & Acer campbellii Hook.f. & Kapase & Tree \\
\hline Aceraceae & Acer sterculiaceum Wall. & Kukurpaile & Tree \\
\hline Actinidiaceae & Actinidia callosa Lindl. & Thekiphal & Herb \\
\hline Amaranthaceae & Cyathula capitata Moq. & & Shrub \\
\hline Anacardiaceae & Rhus javanica Miller & Bhakmilo & Tree \\
\hline Apiaceae & Acronema tenerum (DC.) Edgew. & & Herb \\
\hline Apiaceae & Centella asiatica (L.) Urb. & Ghodtapre & Herb \\
\hline Apiaceae & Heracleum nepalense D. Don & Chimphing & Shrub \\
\hline Apiaceae & Hydrocotyle nepalensis Hook & Ghodtapre & Herb \\
\hline Apiaceae & Selinum tenuifolium Wall. ex C. B. Clarke & & Herb \\
\hline Aquifoliaceae & Ilex dipyrena Wall. & Kande liso & Tree \\
\hline Aquifoliaceae & Ilex excels (Wall.) Voigt & Puwale & Climber \\
\hline Aquifoliaceae & Ilex fragilis Hook.f. & Liso & Tree \\
\hline Araliaceae & Hedera nepalensis K.Koch & Pipal lahara & Climber \\
\hline Araliaceae & Schefflera elata (Buch.-Ham.) Harms & Chinde patta & Shrub \\
\hline Araliaceae & Schefflera impressa (C. B. Clarke) Harms & Bhalu Chide, & Tree \\
\hline Asteraceae & Ageratum conyzoides (L.) L. & Ilamejhar & Herb \\
\hline Asteraceae & Anaphalis adnata Wall. ex DC. & Bukiphool & Herb \\
\hline Asteraceae & Anaphalis contorta (D.Don) Hook.f. & Bukiphool & Herb \\
\hline Asteraceae & Anaphalis margaritacea (L.) Benth. \& Hook. f. & Bukiphool & Herb \\
\hline Asteraceae & Anaphalis triplinervis (Sims) C. B. Clarke & Bukiphool & Herb \\
\hline Asteraceae & Artemisia dubia Wall. & Titepati & Shrub \\
\hline Asteraceae & Aster himalaicus C. B. Clarke & & Herb \\
\hline Asteraceae & Bidens biternata (Lour.) Merr. \& Sherff & kuro & Herb \\
\hline Asteraceae & Bidens pilosa L. & Philungekuro & Herb \\
\hline Asteraceae & Centipeda minima (L.) A. Br. \& Asch. & & Herb \\
\hline Asteraceae & Cirsium verutum (D.Don) Spreng. & & Herb \\
\hline Asteraceae & Cirsium wallichii DC. & Sungur kande & Herb \\
\hline Asteraceae & Dichrocephala benthamii C.B.Clarke & & Herb \\
\hline Asteraceae & Dichrocephala integrifolia (L.f.) Kuntze & Hachheu jhar & Herb \\
\hline Asteraceae & Dubyaea hispida DC. & & Herb \\
\hline Asteraceae & Engeron belidioides & & Herb \\
\hline Asteraceae & Eupatorium adenophorum Spreng. & Banmara & Herb \\
\hline Asteraceae & Galinsoga ciliate (Raf.) S.F.Blake & & Herb \\
\hline Asteraceae & Galinsoga parviflora Cav. & Udase & Herb \\
\hline Asteraceae & Gerbera nivea (DC.) Sch. Bip. & & Herb \\
\hline Asteraceae & Gnaphalium affine D.Don & & Herb \\
\hline Asteraceae & Inula cappa (Buch.-Ham. ex D. Don) DC. & & Shrub \\
\hline Asteraceae & Jurinea dolomitica Galushko & & Herb \\
\hline Asteraceae & Saussurea deltoidea (DC) Sch. Bip. & & Herb \\
\hline Asteraceae & Senecio alatus Wall. ex DC. & Toriphoole & Herb \\
\hline
\end{tabular}


Nepal Journal of Science and Technology Vol. 13, No. 2 (2012) 87-96

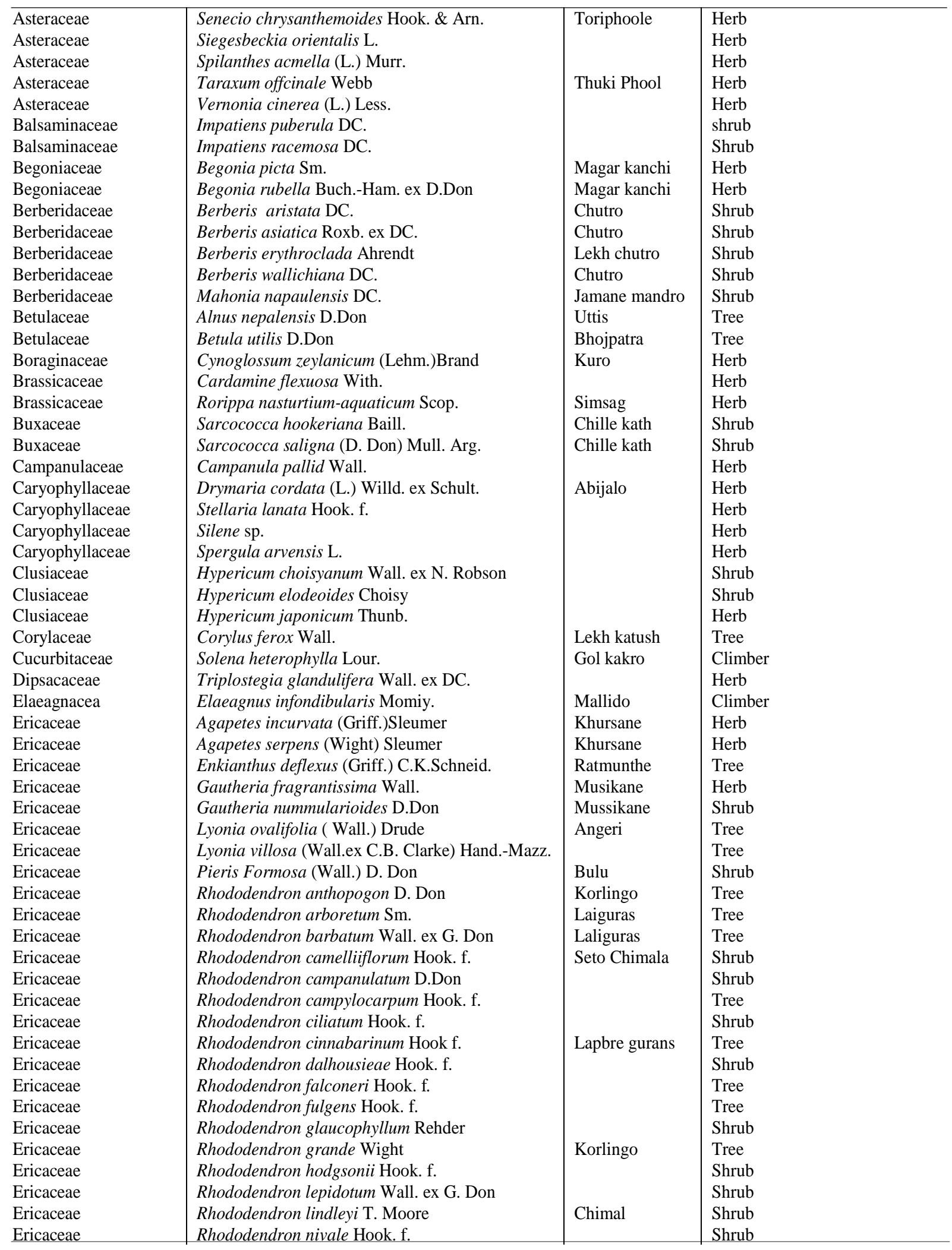


Dilkumar Limbu et al./A checklist of Angiospermic.....

\begin{tabular}{|c|c|c|c|}
\hline Ericaceae & Rhododendron pendulum Hook. f. & & Shrub \\
\hline Ericaceae & Rhododendron pumilum Hook. f. & & Shrub \\
\hline Ericaceae & Rhododendron setosum D. Don & & Shrub \\
\hline Ericaceae & Rhododendron thomsonii Hook. f. & & Shrub \\
\hline Ericaceae & Rhododendron triflorum Hook. f. & & Shrub \\
\hline Ericaceae & Rhododendron vaccinioides Hook. & & Shrub \\
\hline Ericaceae & Rhododendron virgatum Hook. f. & & Shrub \\
\hline Ericaceae & Rhododendron wallichii Hook. f. & & Shrub \\
\hline Ericaceae & Rhododendron wightii Hook. f. & & Shrub \\
\hline Ericaceae & $\begin{array}{l}\text { Vaccinum retusum (Griff.) Hook. f. ex C. B. } \\
\text { Clarke }\end{array}$ & Mussikane & Shrub \\
\hline Ericaceae & Vaccinum vacciniaceum (Roxb.) Sleumer & Sano Mussikane & Shrub \\
\hline Euphorbiaceae & Euphorbia wallichii Hook. f. & Thuki Phool & Herb \\
\hline Euphorbiaceae & Ostodes paniculata Blume & Bepari & Tree \\
\hline Euphorbiaceae & Phyllanthus clarkei Hook. f. & & Herb \\
\hline Fabaceae & Bauhinia variegate L. & Taki & Tree \\
\hline Fabaceae & Erythrina stricta Roxb. & Phaledo & Tree \\
\hline Fabaceae & Parochetus communis D. Don & & Herb \\
\hline Fabaceae & Piptanthus nepalensis (Hook.) D. Don & Tite & Shrub \\
\hline Fabaceae & Trifolium repens L. & & Herb \\
\hline Fagaceae & $\begin{array}{l}\text { Castanopsis hystrix Hook. f. \& Thomson ex A. } \\
\text { DC. }\end{array}$ & Patle Katus & Tree \\
\hline Fagaceae & Castanopsis indica (Roxb. ex Lindl.) A. DC. & Dhalne katus & Tree \\
\hline Fagaceae & Quercus lamellose Sm. & Bajratha & Tree \\
\hline Fagaceae & Quercus lanata Sm. & Bajratha & Tree \\
\hline Fagaceae & Quercus lineate Blume & Phalat & Tree \\
\hline Fagaceae & Quercus semecarpifolia Sm. & Khasru & Tree \\
\hline Gentianaceae & Gentiana ornate (D.Don) Wall. ex Griseb. & & Herb \\
\hline Gentianaceae & Swertia angustifolia Buch.-Ham. ex D. Don & Bhale Chiraito & Herb \\
\hline Gentianaceae & Swertia chirayita (Roxb. ex Fleming) H. Karst. & Chiraito & Herb \\
\hline Gentianaceae & Swertia pedicellata Banerji & Bhale chiraito & Herb \\
\hline Gentianaceae & Tripterospermum volubile (D. Don) H. Hara & & Herb \\
\hline Geraniaceae & Geranium wallichianum D. Don ex Sweet & & Herb \\
\hline Gesnenaceae & Didymocarpus sp. & & Herb \\
\hline Grossulariaceae & Ribes griffithii Hook. f. \& Thomson & & Shrub \\
\hline Hydrangeaceae & Dichroa febrifuga Lour. & & Shrub \\
\hline Juglandaceae & Engelhardia spicata Lechen ex Blume & Mahuwa & Tree \\
\hline Juglandaceae & Juglans regia L. & Okhar & Tree \\
\hline Lamiaceae & Ajuga lobata D. Don & & Herb \\
\hline Lamiaceae & Clinopodium umbrosum (M.Bieb.) Kuntze & & Herb \\
\hline Lamiaceae & Colebrookea oppositifolia Sm. & Dhusure & Shrub \\
\hline Lamiaceae & Elsholtzia blanda (Benth.) Benth. & & Herb \\
\hline Lamiaceae & Elsholtzia strobilifera (Benth.) Benth. & & Herb \\
\hline Lamiaceae & Leucosceptrum canum Sm. & Ghurbis & Tree \\
\hline Lamiaceae & Mentha arvensis L. & Pudina & Herb \\
\hline Lamiaceae & Prunella vulgaris L. & & Herb \\
\hline Lardizabalaceae & Holboelia latifolia Wall. & Guphalo & Herb \\
\hline Lauraceae & $\begin{array}{l}\text { Cinnamomum tamala (Buch.-Ham.) T.Nees \& } \\
\text { Eberm. }\end{array}$ & Sinkauli & Tree \\
\hline Lauraceae & Dodecadnia grandiflora Nees & & Tree \\
\hline Lauraceae & Lindera neesiana (Wall. ex Nees) Kurz & Siltimur & Tree \\
\hline Lauraceae & Lindera pulcherrima (Nees) Hook. f. & Siss & Tree \\
\hline Lauraceae & Litsea monopelata (Roxb.) Pers. & Kutmero & Tree \\
\hline Lauraceae & Neolitsea sp. & Pootele & Tree \\
\hline Lauraceae & Persea glamblei (King \& Hook. f.) Kosterm. & Seto kaulo & Tree \\
\hline Loranthaceae & Viscum album L. & Hadchud & Parasite \\
\hline Magnoliaceae & Magnolia campbellii Hook. f. \& Thomson & Ghoge chap & Tree \\
\hline Magnoliaceae & Michelia dolisopa Buch.-ham. ex DC. & Rani chap & Tree \\
\hline
\end{tabular}




\begin{tabular}{|c|c|c|c|}
\hline Melastomataceae & Melastoma normale D. Don & Chulesi & Shrub \\
\hline Melastomataceae & Osbeckia stellata Buch.-Ham. ex Ker Gawl. & Ashare & Shrub \\
\hline Melastomataceae & Oxyspora paniculata (D. Don) DC. & Bakhra kane & Shrub \\
\hline Melastomataceae & Sonerila tenera Royle & & Herb \\
\hline Meliaceae & Melia azederach L. & Bakaina & Tree \\
\hline Moraceae & Ficus auriculata Lour. & Nebharo & Tree \\
\hline Moraceae & Ficus neriifolia Sm. & Dudhilo & Tree \\
\hline Moraceae & Ficus semicordata Buch.-Ham. ex Sm. & Khaniyu & Tree \\
\hline Moraceae & Morus nigra L. & Kimbu & Shrub \\
\hline Myricaceae & Myrica esculenta Buch.-Ham. ex D. Don & Kaphal & Tree \\
\hline Oleaceae & Osmandthus suavis King ex C.B. Clarke & & Tree \\
\hline Onagraceae & Epilobium roseum (Schreb.) Schreb. & & Herb \\
\hline Onagraceae & Epilobium sikkimense Hausskn. & & Herb \\
\hline Oxalidaceae & Oxalis acetosella $\mathrm{L}$. & Chari amilo & Herb \\
\hline Oxalidaceae & Oxalis corniculata L. & Chari amilo & Herb \\
\hline Papaveraceae & Corydalis cornuta Royle & & Herb \\
\hline Papaveraceae & Meconopsis aculeate Royle & & Herb \\
\hline Phytolaccaceae & Phytolacca acinosa Roxb. & Jaringo & Herb \\
\hline Plantaginaceae & Plantago erosa Wall. & & Herb \\
\hline Plantaginaceae & Plantago major L. & & Herb \\
\hline Polygonaceae & Aconogonum molle (D. Don) H.Hara & Thotne & Herb \\
\hline Polygonaceae & Bistorta amplexicaulis (D. Don) Greene & & Herb \\
\hline Polygonaceae & Persicaria alata (Buch.-Ham. ex D. Don) & Ratnaulo & Herb \\
\hline Polygonaceae & $\begin{array}{l}\text { Persicaria capitata (Buch.-Ham. ex D. Don) H. } \\
\text { Gross }\end{array}$ & Ratnaulo & Herb \\
\hline Polygonaceae & Persicaria chinensis (L.) H. Gross & Ratnaulo & Herb \\
\hline Polygonaceae & Persicaria nepalensis (Meisn.) Miyabe & Ratnaulo & Herb \\
\hline Polygonaceae & Rheum austral D. Don & Padamchal & Herb \\
\hline Polygonaceae & Rumex crispus L. & Halhale sag & Herb \\
\hline Polygonaceae & Rumex nepalensis Spring. & Halhale & Herb \\
\hline Primulacea & Primula calderiana Balf. f. \& Cooper & & Herb \\
\hline Ranunculaceae & Aconitum heterophyllum Wall. ex Royle & Attis & Herb \\
\hline Ranunculaceae & Aconitum spicatum (Bruhl) Stapf & Bikhma & Herb \\
\hline Ranunculaceae & Clematis barbellata Edgew & Jungelahara & Climber \\
\hline Ranunculaceae & Ranunculus hirtellus Royle ex D. Don & & Herb \\
\hline Ranunculaceae & Thalictrum sp. & & Herb \\
\hline Rhamnaceae & Rhamnus napalensis (Wall.) M. A. Lawson & Chille kath & Shrub \\
\hline Rosaceae & Cotoneaster microphyllus Wall. ex Lindl. & Theki Jhar & Shrub \\
\hline Rosaceae & Eriobotrya dubia (Lindl.) Decne. & Jure kaphal & Tree \\
\hline Rosaceae & Fragaria daltoniana J.Gay & Bhui Aiselu & Herb \\
\hline Rosaceae & Fragaria nubicola Lindl. ex Lacaita & Bhui Aiselu & Herb \\
\hline Rosaceae & Geum elatum Wall. ex G. Don & Mula pate & Herb \\
\hline Rosaceae & Geum sikkimense Prain & & Herb \\
\hline Rosaceae & Potentilla fructicosa Hook. f. & Bajradanti & Herb \\
\hline Rosaceae & Potentilla fulgens Wall. ex Hook. & Bajradanti & Herb \\
\hline Rosaceae & Potentilla lineate Trev. & Bajradanti & Herb \\
\hline Rosaceae & Prunus cerasoides D.Don & Paiyu & Tree \\
\hline Rosaceae & Prunus cornuta (Wall. ex Royle) Steud & Arupate & Tree \\
\hline Rosaceae & Pyracantha crenulata (D. Don) M. Roem. & Ghangaru & Shrub \\
\hline Rosaceae & Pyrus pashia Buch.-Ham. ex D. Don & Mehel & Tree \\
\hline Rosaceae & Rosa sericea Lindl. & JangaliGulab & Shrub \\
\hline Rosaceae & Rubus accuminatus Sm. & Rato Aiselu & Shrub \\
\hline Rosaceae & Rubus biflorus Buch.-Ham. ex Sm. & & Climber \\
\hline Rosaceae & Rubus ellipticus Sm. & Pahelo Aiselu & Shrub \\
\hline Rosaceae & Rubus foliolosus D. Don & kalo Aiselu & Climber \\
\hline Rosaceae & Rubus paniculatus Sm. & Rukha Aiselu & Climber \\
\hline Rosaceae & Sorbus sp. & & Shrub \\
\hline Rubiaceae & Galium acutum Edgew. & & Herb \\
\hline
\end{tabular}




\begin{tabular}{|c|c|c|c|}
\hline Rubiaceae & Hymenopogon parasiticus Wall. & Phirphire & Epiphyte \\
\hline Rubiaceae & Rubia manjith Roxb. ex Fleming & Majitho & Climber \\
\hline Rutaceae & Euodia fraxinifolia (D. Don) Hook. f. & Khanakpa & Tree \\
\hline Rutaceae & Skimmia sp. & Gandhe & Tree \\
\hline Rutaceae & Zanthoxylum armatum DC. & Timur & Shrub \\
\hline Salicaceae & Salix psilostigma Anderson & Bais & Tree \\
\hline Sambucaceae & Sambucus adnata Wall. ex DC. & Chanbhang & Herb \\
\hline Sambucaceae & Viburnum cotinifolium D. Don & Baklo pate & Shrub \\
\hline Sambucaceae & Viburnum cylindricum Buch.-Ham. ex D. Don & Ghodakhori & Tree \\
\hline Sambucaceae & Viburnum erubescens Wall. ex DC. & Asare & Shrub \\
\hline Sambucaceae & Viburnum mullaha Buch.-Ham. ex D. Don & Kabase & Shrub \\
\hline Sambucaceae & Viburnum nervosum D. Don & Bhale gogan & Shrub \\
\hline Santalaceae & Osyris wightiana Wall. ex Wight & Nundhiki & Shrub \\
\hline Santalaceae & Pyrularia edulis (Wall. ex Roxb.) & Amphi & Tree \\
\hline Saururaceae & Houttuynia cordata Thunb. & Gardhe & Herb \\
\hline Saurauiaceae & Saurauia nepaulensis DC. & Gogan & Tree \\
\hline Saxifragaceae & Astilbe rivularis Buch.-ham. ex D. Don & Budho okhati & Herb \\
\hline Saxifragaceae & Bergenia ciliate (Haw.) Sternb. & Pakhanved & Herb \\
\hline Saxifragaceae & Saxifraga brachypoda D. Don & & Herb \\
\hline Saxifragaceae & Saxifraga diversifolia Wall. ex Ser. & & Herb \\
\hline Saxifragaceae & Saxifraga duthiei Grand. & & Herb \\
\hline Schisandraceae & $\begin{array}{l}\text { Schisandra grandiflora (Wall.) Hook. f. \& } \\
\text { Thomson }\end{array}$ & Sigauto & Climber \\
\hline Scrophulariaceae & Hemiphragma heterophyllum Wall. & & Herb \\
\hline Scrophulariaceae & Mazus japonicusm (Thunb.) Kuntze & & Herb \\
\hline Scrophulariaceae & Mazus surculosus D. Don & & Herb \\
\hline Scrophulariaceae & $\begin{array}{l}\text { Neopicrorhiza scrophulariiflora (Pennell) D. Y. } \\
\text { Hong }\end{array}$ & Kudki & Herb \\
\hline Solanaceae & Solanum aculeatissimum Jacq. & & Herb \\
\hline Symplocaceae & Symplocos pyrifolia Wall. ex G. Don & Kholme & Tree \\
\hline Symplocaceae & Symplocos ramosissima Wall. ex G. Don & & Tree \\
\hline Symplocaceae & Symplocos theifolia (Hayata) Hayata & Bakalapate & Tree \\
\hline Theaceae & Camellia kissi Wall. & Hinguwa & Shrub \\
\hline Theaceae & Eurya acuminate DC. & Jhinganu & Tree \\
\hline Theaceae & Eurya sp. & Thulo Jhigano & Shrub \\
\hline Theaceae & Schima wallichii (DC.) Korth. & Chilaune & Tree \\
\hline Thymelaeaceae & Daphne bholua Buch.-ham. ex D. Don & Lokta & Shrub \\
\hline Thymelaeaceae & Daphne papyracea Wall. ex Steud. & Lokta & Shrub \\
\hline Thymelaeaceae & Edgeworthia gardneri (Wall.) Meisn. & Argeli & Shrub \\
\hline Urticaceae & Boehmeria platyphylla D. Don & Gargalo & Herb \\
\hline Urticaceae & Elatostema platyphyllum Wedd. & & Herb \\
\hline Urticaceae & Girardinia diversifolia (Link) Friis & Allo & Shrub \\
\hline Urticaceae & Gonostegia hirta (Blume) Mig. & Chiple & Herb \\
\hline Urticaceae & Laportea terminalis Wight & Patle Sisnu & Herb \\
\hline Urticaceae & Leucanthus peduncularis (Wall. ex Royle) Wedd. & Gagleti & Herb \\
\hline Urticaceae & Pilea umbrosa Blume & & Herb \\
\hline Urticaceae & Urtica dioica $\mathrm{L}$. & Sisnu & Herb \\
\hline Valerianaceae & Valeriana hardwickii Wall. & & Herb \\
\hline Valerianaceae & Valeriana jatamansii Jones & Sugandhawal & Herb \\
\hline Violaceae & Viola biflora $\mathrm{L}$. & & Herb \\
\hline Violaceae & Viola pilosa Blume & & Herb \\
\hline Vitaceaee & Parthenocissus himalayana (Royle) Planch. & & Climber \\
\hline Monocotyledon & & & \\
\hline Alliaceae & Allium hypsistum Stearn & Jimbu & Herb \\
\hline Alliaceae & Allium wallichii Kunth & Dungdunge & Herb \\
\hline Amaryllidaceae & Zephyranthes carinata Herb. & Ban lasun & Herb \\
\hline Araceae & Acorus calamus L. & Bojho & Herb \\
\hline Araceae & Arisaema concinnum Schott & Bako & Herb \\
\hline
\end{tabular}


Nepal Journal of Science and Technology Vol. 13, No. 2 (2012) 87-96

\begin{tabular}{|c|c|c|c|}
\hline Araceae & Arisaema erubescens (Wall.) Schott. & Bako & Herb \\
\hline Araceae & Arisaema griffithii Schott. & Sap ko makai, & Herb \\
\hline Araceae & Arisaema intermedium Blume & Bako & Herb \\
\hline Araceae & Arisaema nepenthoides (Wall.) Mart. ex Schott. & Bako & Herb \\
\hline Araceae & Arisaema propinquum Schott. & Sap ko makai & Herb \\
\hline Liliaceae & Asparagus racemosus Wall. & Kurilo & Climber \\
\hline Commelinaceae & Cyanotis axillaris (L.) D. Don ex Sweet & & Herb \\
\hline Commelinaceae & Commelina paludosa Blume & Kane & Herb \\
\hline Суреraceae & Carex foliosa D. Don & Harkato & Herb \\
\hline Сyperaceae & Carex myosurus Nees & Harkato & Herb \\
\hline Суреraceae & Carex nubigena D. Don & Harkato & Herb \\
\hline Суреraceae & Carex rara $\mathrm{B}$. & Harkato & Herb \\
\hline Суреraceae & Carex rochebrunii Franch. ex Sav. & Harkato & Herb \\
\hline Суреraceae & Carex setigera D. Don & Harkato & Herb \\
\hline Сурегасеае & Fimbristylis aestivalis (Retz.) Vahl & & Herb \\
\hline Сyperaceae & Fimbristylis dichotomam (L.) Vahl. & & Herb \\
\hline Cyperaceae & Kobresia sp. & & Herb \\
\hline Сyperaceae & $\begin{array}{l}\text { Pycreus sanguinolentus (Vahl) Nees ex C. B. } \\
\text { Clarke }\end{array}$ & & Herb \\
\hline Dioscoreaceae & Dioscorea bulbifera L. & Bhyagur & Climber \\
\hline Dioscoreaceae & Dioscorea esculenta (Lour.) Burkill & Ban tarul & Climber \\
\hline Iridaceae & Amomum subulatum Roxb. & Alainchi & Herb \\
\hline Juncaceae & Juncus leucanthus Royle ex D. Don & & Herb \\
\hline Juncaceae & Juncus ochraceus Buchenau & & Herb \\
\hline Juncaceae & Juncus wallichianus Laharpe & & Herb \\
\hline Juncaceae & Luzula plumose E. May. & & Herb \\
\hline Liliaceae & Campylandra aurantiaca Baker & & Herb \\
\hline Liliaceae & $\begin{array}{l}\text { Clintonia udensis var. alpine Trautv. \& C. A. } \\
\text { Mey. }\end{array}$ & & Herb \\
\hline Liliaceae & Lillium nepalense D. Don & Lili & Herb \\
\hline Liliaceae & Paris polyphylla (Stearn) H. Hara & Satuwa & Herb \\
\hline Liliaceae & Polygonatum verticillatum (L.) All. & Khiraula & Herb \\
\hline Liliaceae & Smilacina oleracea (Bekar) Hook. f. & Khiraula & Herb \\
\hline Liliaceae & Smilacina purpurea Wall. & Khiraula & Herb \\
\hline Orchidaceae & Coelogyne corymbosa Lindl. & & Herb \\
\hline Orchidaceae & Coelogyne punctulata Lindl. & Sunkhari & Herb \\
\hline Orchidaceae & Dactylorhiza hatagirea (D. Don) Soo & Pancha aule & Herb \\
\hline Orchidaceae & Pleione hookeriana (Lindl.) J. Moore & Sunkhari & Epiphyte \\
\hline Orchidaceae & Pleione humilis (Sm.) D. Don & Sunkhari & Epiphyte \\
\hline Orchidaceae & Satyrium nepalense D. Don & & Herb \\
\hline Orchidaceae & Spiranthes sinensis (M. Bieb.) H. Hara & & Herb \\
\hline Poaceae & Agrostis pilosula Trin. & & Herb \\
\hline Poaceae & Arundinaria maling Gamble & Malingo & Herb \\
\hline Poaceae & Arundo donax L. & & Herb \\
\hline Poaceae & Capillipedium assimile (Steud.) A. Camus & Kharuki & Herb \\
\hline Poaceae & Cynodon dactylon (L.) Pers. & Dubo & Herb \\
\hline Poaceae & Danthonia schneideri Pilg. & & Herb \\
\hline Poaceae & Digitaria cruciata (Nees ex Steud.) A. Camus & Banso & Herb \\
\hline Poaceae & Eragrostis nigra Nees ex steud. & Banso & Herb \\
\hline Poaceae & Eulalia mollis (Griseb.) Kuntze & & Herb \\
\hline Poaceae & Helictotrichon parviflorum (Hook. f.) Bor & & Herb \\
\hline Poaceae & Imperata cylindrical (L.) P. Beauv. & Siru & Herb \\
\hline Poaceae & Isachne miliacea Roth & & Herb \\
\hline Poaceae & Microstegium ciliatum (Trin.) A. Camus & & Herb \\
\hline Poaceae & Pennisetum cladistrum (Trin.) A. Camus & Kiku & Herb \\
\hline Poaceae & Poa annua L. & Kode & Herb \\
\hline Poaceae & Setaria pumila (Poir.) Roem. \& Schult. & & Herb \\
\hline
\end{tabular}


Dilkumar Limbu et al./A checklist of Angiospermic.....

\begin{tabular}{l|l|l|l}
\hline Poaceae & Thamnocalamus spathiflorus (Trin.) Munro & Nigalo & Shrub \\
Poaceae & Thysanolaena maxima (Roxb.) Kuntze & Amliso & Shrub \\
Smilacaceae & Smilax aspera L. & Kukurdaino & Climber \\
Zingiberaceae & Hedychium spicatum Sm. & Saro & Herb \\
Zingiberaceae & Roscoea purpurea Sm. & Bhui saro & Herb \\
\hline
\end{tabular}

The collected plants are not alpine flora because this work was carried out below $4100 \mathrm{~m}$ asl altitude in Tinjure-Milke-Jaljale area. On the other hand, Japanese expedition teams had collected about 578 species of angiospermic alpine flora in 1972, 1977 and 1991 from alpine region of Jaljale Himal (Ohba \& Akiyama 1992). There are only 72 species out of 326 species collected plants are re-reported in this work from the alpine flora of the Jaljale Himal.

Majority of the plants are herbaceous, there are 178 species of herbs, 67 species of shrubs, 62 species of trees, 15 species of climbers and other 4 species (Fig. 2). This work is not a complete report of angiospermic plants from Tinjure-Milke-Jaljale area. It needs more intense plant collection for a complete report.

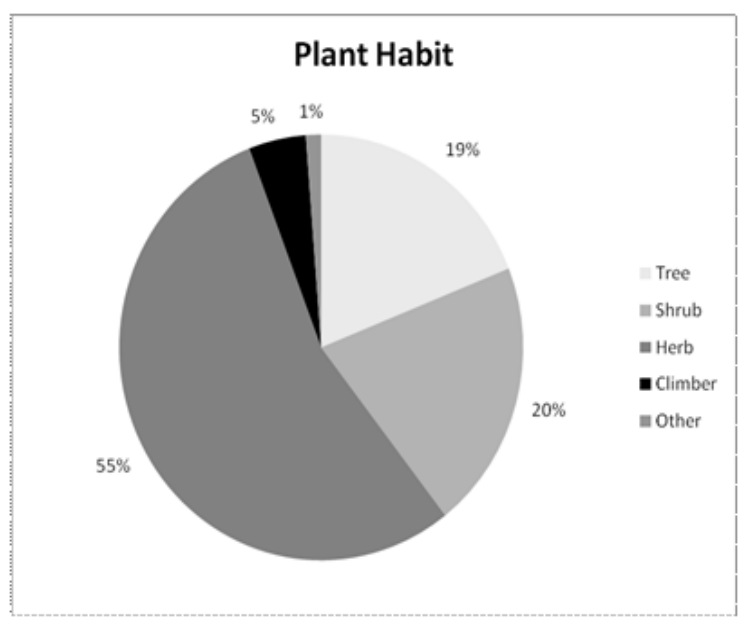

Fig. 2. Graphic presentation of pant's habit of TMJ

\section{Acknowledgements}

We would like to thank Mr. J.B. Limbu and Mr. M. Wongdi Sherpa who accompanied us for plants collection in Milke-Jaljale area. Similarly, we are very thankful to the personnel of National Herbarium and Plant Laboratories, Godawari, Lalitpur, Nepal for plant identification. One of the authors (Limbu) is grateful to the University Grants Commission, Nepal for the research fellowship.

\section{References}

Chaudhari, R.P. and R.S.L. Taylor. 2000. Eco-tourism in the Rhododendron hotspot of Nepal. Welcome Nepal 8(2): 5-12.

Hara, W., W.T. Stearn and L.H., Williams. 1978. An enumeration of the flowering plants of Nepal, vol. 1. British Museum (Nat.Hist.), London.

Hara, W. and L.H. Williams. 1979. An enumeration of the flowering plants of Nepal, vol. 2. British Museum (Nat.Hist.), London.

Hara, W., A.O. Chater and L.H. Williams. 1982. An enumeration of the flowering plants of Nepal, vol. 3. British Museum (Nat.Hist.), London.

IUCN. 2002. An assessment of Tinjure-Milke-Jaljale (TMJ) area of eastern Nepal. (Ed. Krishna P. Oli) International Union for Conservation of Nature (IUCN), Nepal

Koirala, M. and D.K. Limbu. 2010. Development of new protected area project, Tinjure-Milke-Jaljale Area. National Trust for Nature Conservation (NTNC), Lalitpur, Nepal.

Limbu, D.K. and M. Koirala. 2011. Above-ground and below-ground biomass situation of Milke-Jaljale Rangeland at Different Altitudinal Gradient. Our Nature 9:107 - 111.

MOPE .1997. National rhododendron environmental conservation area. Ministry of Population and Environment, Kathmandu, Nepal.

Milleville, R. 2002. The Rhododendrons of Nepal. Himal Books, Patan Dhoka, Nepal.

Ohba, H. and S. Akiyama. 1992. The alpine flora of the Jaljale Himal, East Nepal. The University Museum, the University of Tokyo, Nature and Culture No. 4.

Polunin, O. and A. Stainton 2003. Flowers of the Himalaya. Oxford University Press, New Delhi.

Press, J.R., K.K. Shrestha and D.A. Sutton. Annotated checklist of the flowering plants of Nepal. www.Efloras.org

Press, J.R., K.K. Shrestha and D.A. Sutton 2000. Annotated checklist of the flowering plants of Nepal. The natural History Museum, London.

Primack, R.B. 1998. Essentials of conservation biology. Boston University, Sunderland, Massachusetts, U.S.A.

Stainton, A. 2003. Flowers of the Himalaya a Supplement. Oxford University Press, New Delhi, India.

Watson, M.F. et al. 2011. Flora of Nepal Vol. 3. Royal Botanical Garden Endinburgh.

The Plant List, 2010. Version 1. Published on the Internet; http://www.theplantlist.org/ (accessed 7 September 2012) 
Nepal Journal of Science and Technology Vol. 13, No. 2 (2012) 87-96 